\title{
Direct Insertion of a Short-Type Single-Balloon Enteroscope and Using a Stent Retriever to Treat Difficult Bile Duct Stones in Surgically Altered Anatomy 四
}

\author{
Takashi Sasaki and Naoki Sasahira \\ Department of Hepato-Biliary Pancreatic Medicine, Cancer Institute Hospital of Japanese Foundation for Cancer Research, Tokyo, \\ Japan
}

A 78-year-old man who underwent distal gastrectomy with Roux-en-Y reconstruction for gastric cancer presented with multiple bile duct stones. A short-type (length, $152 \mathrm{~cm}$ ) single-balloon-enteroscope (SBE) with a $3.2 \mathrm{~mm}$ working channel (SIF-H290S; Olympus Medical, Tokyo, Japan) was used for balloon enteroscopy-assisted endoscopic retrograde cholangiopancreatography (Fig. 1A). Small endoscopic sphincterotomy combined with endoscopic papillary large balloon dilation (EPLBD) $(15 \mathrm{~mm})$ was performed (Fig. 1B), and small stones were easily extracted. However, a large, impacted stone remained in the common bile duct. A short-type SBE (outer diameter $9.2 \mathrm{~mm}$ ) was inserted into the common bile duct via the dilated papilla (Fig. 2A, Supplementary Video 1). The impacted stone was directly visualized and was crushed using a 7 Fr stent retriever (Fig. 2B). Then, small pieces of the stones were successfully removed using a basket catheter and retrieval balloon without complications (Fig. 2C).

Difficult bile duct stone removal is not uncommon. ${ }^{1} \mathrm{~A}$ balloon enteroscope is useful for stone removal in patients with surgically altered anatomy. ${ }^{2}$ In cases of large impacted stones, cholangioscopy-assisted lithotripsy is often necessary, but pro- cedures using both SBE and cholangioscope are demanding., ${ }^{3,4}$ As an alternative method, we inserted a SBE into the common bile duct. ${ }^{5,6}$ There are a few technical tips to perform this procedure safely. First, the papilla needs to be dilated with EPLBD to enable the insertion of SBE into the common bile duct. Second, we used a stent retriever to crush the impacted stones. Although electronic hydraulic or laser lithotripsy are useful devices for impacted stone removal, they are expensive and have limited availability. On the other hand, a stent retriever is cheap and available in most institutions. Third, visualization of impacted stone is critical for safety. Even when the SBE cannot pass the papilla, repetitive insertion of a stent retriever over the guidewire can crush the impacted stone and make space for subsequent use of a basket catheter. As there is a risk of inflicting bile duct injury or insufficient crushing of impacted stones, it is better to visualize the impacted stone directly then crush it with a stent retriever. In this regard, the use of a short-type SBE combined with a stent retriever is a reasonable approach for the removal of impacted bile duct stones in patients with surgically altered anatomy.

Received: May 31, 2020 Revised: June 11, 2020

Accepted: June 16, 2020

Correspondence: Takashi Sasaki

Department of Hepato-Biliary Pancreatic Medicine, Cancer Institute Hospital of Japanese Foundation for Cancer Research, 3-8-31, Ariake, Koto, Tokyo 135-8550, Japan

Tel: +81-3-3520-0111, Fax: +81-3-3520-0141, E-mail: sasakit-tky@umin.ac.jp

ORCID: https://orcid.org/0000-0001-7109-9835

(c) This is an Open Access article distributed under the terms of the Creative Commons Attribution Non-Commercial License (http://creativecommons.org/licenses/by$\mathrm{nc} / 3.0$ ) which permits unrestricted non-commercial use, distribution, and reproduction in any medium, provided the original work is properly cited. 


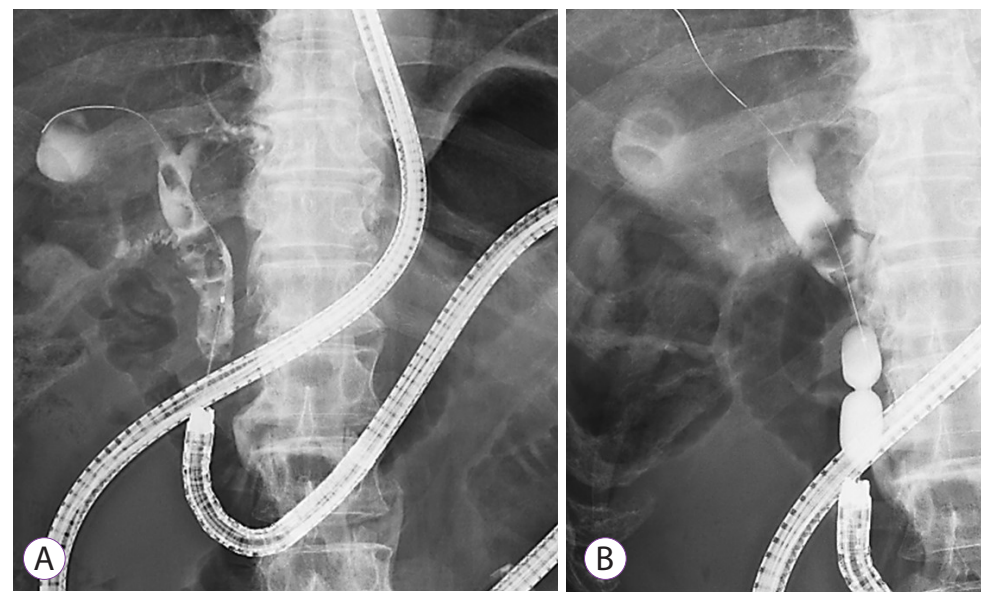

Fig. 1. (A) Multiple bile duct stones were identified in the common bile duct. (B) Endoscopic papillary large balloon dilation with $15 \mathrm{~mm}$ was performed.
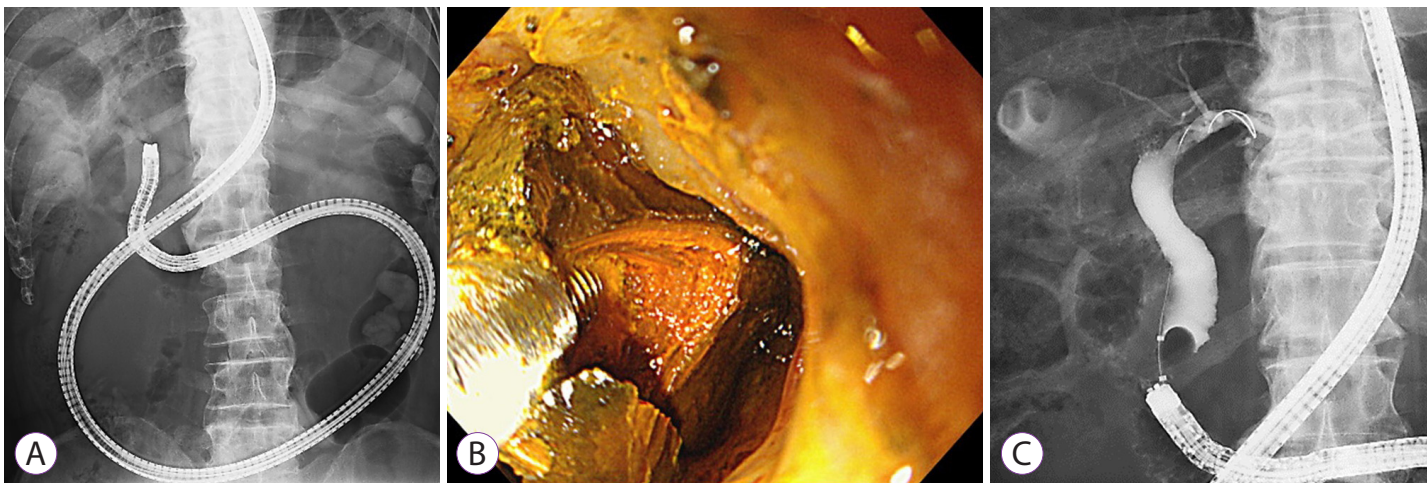

Fig. 2. (A) Direct insertion of a short-type single-balloon-enteroscope for cholangioscopy. (B) The impacted stone was crushed using a $7 \mathrm{Fr}$ stent retriever under direct view. (C) Multiple bile duct stones were successfully extracted.

Conflicts of Interest

The authors have no potential conflicts of interest.

Funding

None.

Author Contributions

Data curation: Takashi Sasaki, Naoki Sasahira

Writing-original draft: TS

Writing-review\&editing: NS

ORCID

Takashi Sasaki:

Naoki Sasahira:

https://orcid.org/0000-0001-7109-9835 https://orcid.org/0000-0002-0672-7214

\section{Supplementary Material}

Video 1. Direct insertion of a short-type single-balloon-enteroscope for cholangioscopy and using a stent retriever to treat difficult bile duct stones in surgically altered anatomy (https://doi.org/10.5946/ce.2020.145.v001).

\section{REFERENCES}

1. Nakai Y, Sato T, Hakuta R, et al. Management of difficult bile duct stones by large balloon, cholangioscopy, enteroscopy and endosonography. Gut Liver 2020;14:297-305.

2. Soh JS, Yang DH, Lee SS, et al. Single balloon enteroscopy-assisted endoscopic retrograde cholangiopancreatography in patients who underwent a gastrectomy with Roux-en-Y anastomosis: six cases from a single center. Clin Endosc 2015;48:452-457.

3. Tonozuka R, Itoi T, Sofuni A, et al. Novel peroral direct digital cholangioscopy-assisted lithotripsy using a monorail technique through the overtube in patients with surgically altered anatomy (with video). Dig Endosc 2019;31:203-208.

4. Mandai K, Uno K, Yasuda K. Efficacy and safety of short-type single balloon enteroscopy-assisted peroral direct cholangioscopy using an ultra-slim endoscope in patients with surgically altered anatomy. Surg Laparosc Endosc Percutan Tech 2020;30:327-331.

5. Yamauchi H, Kida M, Miyazawa S, Okuwaki K, Imaizumi H, Koizumi W. Electrohydraulic lithotripsy under peroral direct cholangioscopy using short-type single-balloon enteroscope for large common bile duct stone in patients with Roux-en-Y gastrectomy. Endoscopy 2015;47(Suppl 1):E240-E241.

6. Yamauchi H, Kida M, Okuwaki K, et al. Therapeutic peroral direct cholangioscopy using a single balloon enteroscope in patients with Rouxen-Y anastomosis (with videos). Surg Endosc 2018;32:498-506. 